\title{
Measurement of Luminous Intensity Using Filtered Trap Detector
}

\author{
M. S. Khali1 ${ }^{1}$, F. M. E-Sharkawy ${ }^{1, *}$, M. G. EL- Shaarawy ${ }^{2}$, N. Shash ${ }^{2}$, A. Mahmoud ${ }^{1}$ \\ ${ }^{1}$ Photometry Department; National Institute of Standards, Giza, Egypt \\ ${ }^{2}$ Physics Department; Faculty of sciences, Benha, University
}

\begin{abstract}
The work described in this paper is a part of the process of lamp and photometer calibration to obtain a new luminous-intensity scale with a lower uncertainty. The procedure and the results of realizing the photometric scale with a standardized detector at the National Institute for Standards (NIS) are described. This method enables photometric values to be derived from absolute spectroradiometric measurements by exact computation. A filtered trap detector has been characterized and used to measure the absolute spectral power distribution of standard la mps. From these data a photometric scale of lu minous intensity has been realized. The results were compared to the certified calib ration values obtained by National Physical Laboratory (NPL), and showed agreement within uncertainty of $\pm 1.8 \%$
\end{abstract}

Keywords Photometry, Spectral Irradiance, Trap Detector, Luminous Intensity, Filtered Radio meter

\section{Introduction}

The photometric base unit of the International System of Units (SI) is the candela (cd), the unit of luminous intensity. Since 1979, the candela has been defined as: the luminous intensity, in a given direction, of a source that emits monochromatic radiation of frequency $540 \times 10^{12}$ hertz and that has a radiant intensity in that direction of (1/683) watt per steradian $^{[1-4]}$. The definition of the candela links together photometric and radiometric quantities. The basis of accurate photometric evaluations of light sources is provided by accurate spectral measurements under specified geometric conditions.

The National Institute of Standards in Egypt (NIS) used to maintain the luminous intensity by a group of specially designed lamps periodically calibrated at NPL (National Physical Laboratory- England), with uncertainty of $\pm 0.8 \%$ However the scale can be realized by using detector-based methods. Owing to recent improvements in radiometric measurements, units for most photometric quantities can now be realized with the highest accuracy by radiometric methods. As trap detectors have been extensively described by other authors ${ }^{(5-8)}$. Their working princip le is summarized only briefly here. Irradiance standard lamps can be accurately calibrated in absolute values using the trap detector and set of interference filters. As

$$
E_{v}=K_{m} \int_{380}^{780} E(\lambda) V(\lambda) d \lambda
$$

* Corresponding author:

fsharkawy_2000@yahoo.com (F. M. E-Sharkawy)

Published online at http://journal.sapub.org/optics

Copyright (C) 2012 Scientific \& Academic Publishing. All Rights Reserved

$$
\mathbf{I}=\mathbf{E}_{\mathbf{v}} \mathbf{d}^{2}
$$

Where $\mathbf{E}_{\mathbf{v}}$ is the illuminance, $\mathbf{I}$ is the luminous intensity in candelas, and $\mathbf{d}$ is the distance between the light source and the irrad iated surface.

The photometric unit can be derived from the spectral irradiance distribution by means of a $v$ isual response function $\mathbf{V}(\boldsymbol{\lambda})$ defined and tabulated by the CIE and the constant factor $\mathbf{K}_{\mathbf{m}}$ specified in the new defin ition.

\section{Basis of the Measurement}

The spectral irrradiance of a tungsten lamp $\mathbf{E}(\boldsymbol{\lambda})$ at a distance $d$ as a function of $\lambda$, is defined as :

$$
\mathbf{E}(\lambda)=\frac{\varphi(\lambda)}{A}
$$

Where $\varphi(\lambda)$ radiant flux incident on an aperture area A. Depending on the optical radiation incident on the effective aperture area, filter radio meter generates photocurrent as

$$
\Gamma_{c i}=\int_{A} \varphi(\lambda) \tau(\lambda) R(\lambda) d \lambda
$$

Where $\tau(\lambda)$ is the spectral transmittance of the filters, $\mathbf{R}(\lambda)$ is the known spectral responsivity of the trap detector, and $\lambda$ is the radiation wavelength in air. Since the spectrum of light source used in measurements is close to Planck radiator, the spectral power distribution of the light source in eq. (3) for over the visible range is estimated as given in the following analytical form:

$$
\varphi(\lambda)=\frac{A_{3} \cdot \lambda^{3}+A_{2} \cdot \lambda^{2}+A_{1} \cdot \lambda+A_{0}}{\exp (B / A) \cdot \lambda^{5}}
$$

Where $\boldsymbol{A}_{0}, \boldsymbol{A}_{1}, \boldsymbol{A}_{2}, \boldsymbol{A}_{3} \& \boldsymbol{B}$ are free parameters. The spectral 
irradiance was modeled with 3rd degree of polynomial to compensate for deviations of the lamp used in measurement fro $m$ that of ideal b lackbody radiator. ${ }^{(9-10)}$

From eq. (4) \& eq. (3) we can obtain the following equation for the various filters as

$$
I_{c, i}=\int_{A} \frac{A_{3} \cdot \lambda^{3}+A_{2} \cdot \lambda^{2}+A_{1} \cdot \lambda+A_{0}}{\exp (B / A) \cdot \lambda^{5}} \tau(\lambda) R(\lambda) d \lambda
$$

where $\boldsymbol{i}$ indicates the filters which are used in the measurements. Equation (4) must usually be solved by an iterative method. We have to minimize the relative errors between the measured and calculated values of the photo currents $\boldsymbol{I}_{\boldsymbol{m}, \boldsymbol{i}} \&$ $\boldsymbol{I}_{c, i}$ to minimum using the least square fitting:

$$
\begin{aligned}
\text { Error }\left(A_{0}, A_{1}, A_{2}, A_{3} \text { and } B\right) & =\sum_{i=1}^{7}\left(\frac{I_{m, i}-I_{C, i}}{I_{m, i}}\right)^{2} \\
& =\text { minimum }
\end{aligned}
$$

(for the seven filters).

The detailed explanation for the solutions of these type equations can be found in ref.[11]. The luminous intensity of the lamp can be calculated from spectral irradiance and the luminous efficacy function, $\mathbf{V}(\boldsymbol{\lambda})$ from equation (1)

\section{Experimental Technique.}

\subsection{Filter Radi ometer}

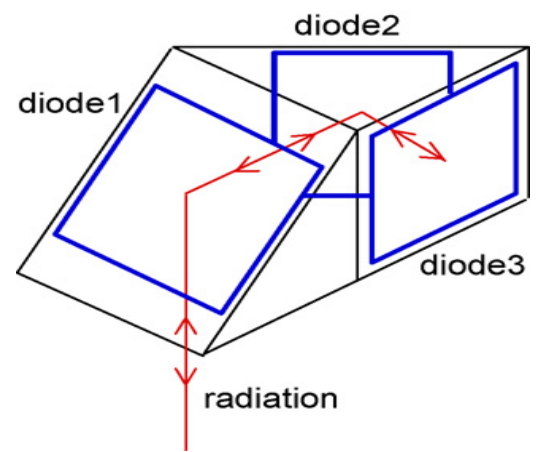

Figure 1. Schematic representation of the trap detector tector, interference filters and precision aperture. The trap detector consists of three windowless Hamamatsu 13371 type silicon photodiodes; each has $1 \mathrm{~cm}^{2}$ active area. In trap detector design, the photodiodes are geometrically arranged in such a way that polarization sensivity is removed. In this design, incident beam undergoes five reflections before leaving the detector as shown in Fig. 1. Therefore, reflectance of a trap detector is nearly zero. A precision circular aperture of area $0.1 \mathrm{~cm}$ was attached to the front of the trap detector.

Application of a bias voltage increases the linearity limit by a factor more than 5

\subsection{Filter Tr ans mittance}

Seven interference filters from Avian, having nominal central wavelengths at $400 \mathrm{~nm}, 450 \mathrm{~nm}, 500 \mathrm{~nm}, 550 \mathrm{~nm}$, 600 , and $650 \mathrm{~nm}$ and $700 \mathrm{~nm}$, were used for the measurements. All filters have a path band of $10 \mathrm{~nm}$. The spectral transmittances of the filters were measured with a Shimadzu double monochromator with $0.1 \mathrm{~nm}$ interval and band slit width $0.3 \mathrm{~nm}$ (Fig.2). Before starting transmittance measurements the wavelength calibration of monochromator was performed. The filters were checked out of the path band. It blocks radiation away from the band path region to better than $0.01 \%$ -

\section{Measurement Set-Up}

Two set of lamps, 1000 watt standard FEL type and Osram 200 watt $\mathrm{Wi} / \mathrm{G}$ lamps were used as light sources having high optical radiation during measurements. Lamps were operated with DC power at fixed polarity and at a stabilized lamp current. The lamp current was measured as the voltage across a calibrated reference resistor of $0.1 \mathrm{Ohm}$ using DVM Fluke 8085 . The photocurrent of the photometer was measured using a calibrated digital LMT nano-photocurrent meter I1000. Baffles were employed to minimize the stray-light.

The filter radiometer is basically consists of the trap de-

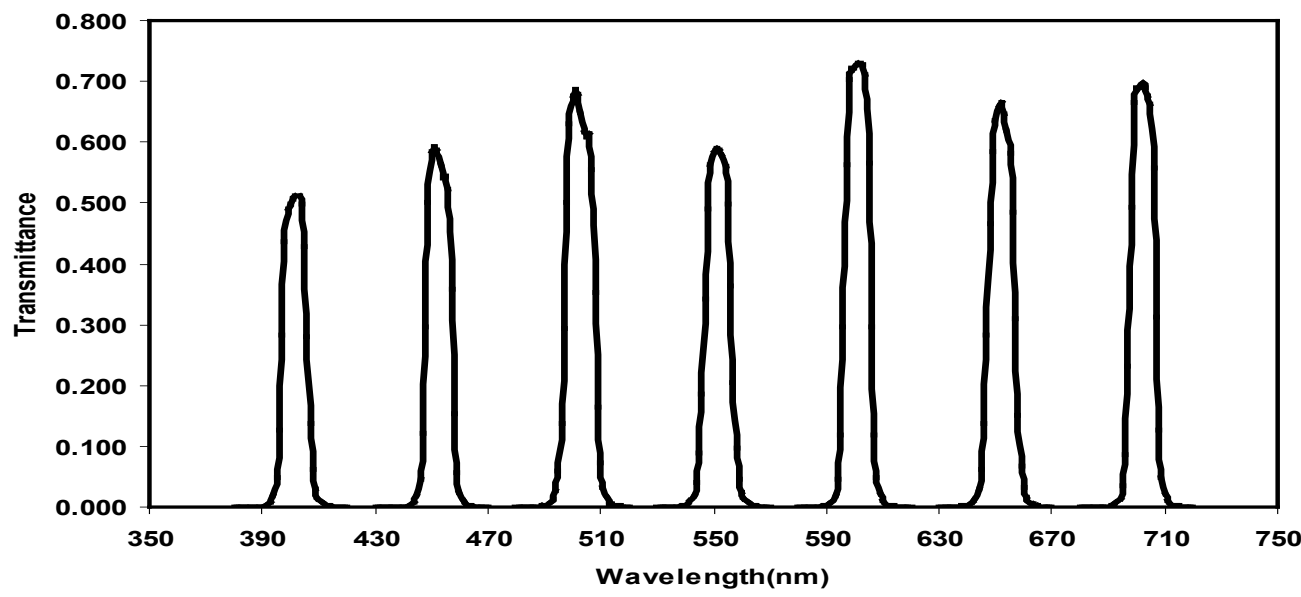

Figure 2. Spectraltransmittance of the filters 


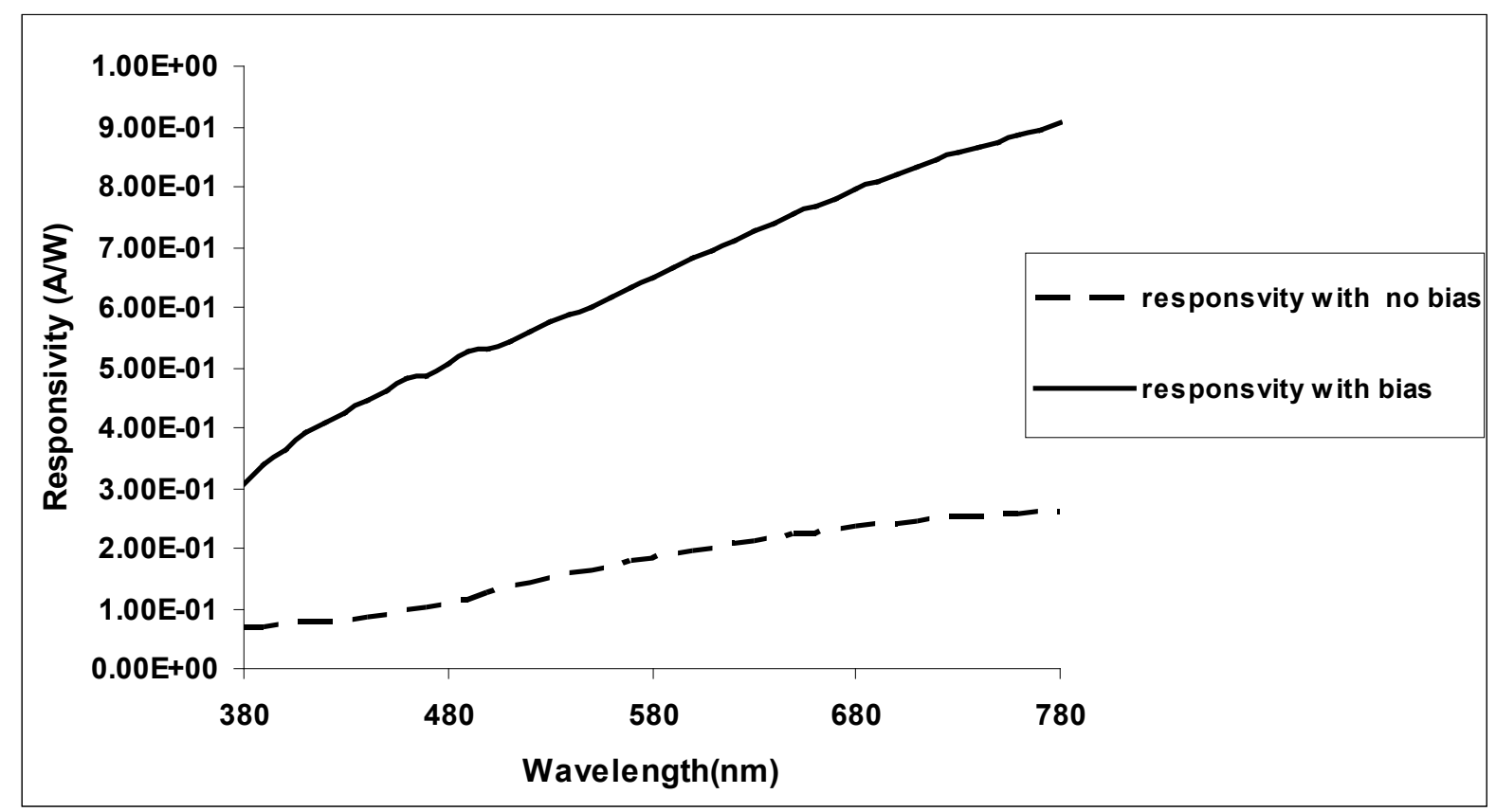

Figure 3. Responsivity of trap detector

The trap detector and the baffles were placed on 4.5 meter optical bench equipped with an accurate length scale. The lamps were mounted on stages with five degrees of freedom that allow accurate positioning of the lamp filament. The optical axis of the bench was determined with an alignment laser positioned between the detector and the lamp. The detector to lamp distance was measured from the lamp filament plane to the plane of the aperture. Filter radio meter was used to measure the irradiance values on the aperture plane of the trap detector. e transmittance range of the filters.

\subsection{Spatial Unifor mity}

The response uniformity of the active area of light- trapping detector was investigated. Nonuniformity would produce measurement errors when the detector used in different position. The uncertainty due to response nonuniformity depends on the position and size of the beam ${ }^{[14-15]}$. The measurements were done by using a Helium-Neon laser. The detector was moved in $\mathrm{x}$ and $\mathrm{y}$ axis and the output radiation were scanned across the surface.

\section{Characterization of the Trap Detector}

\subsection{Spectral Responsivity of the Detector}

The spectral responsivities of the polarization independent reflection trap detector, was determined in the range 350 $-800 \mathrm{~nm}$ (Fig. 3). Using a high-accuracy spectrophotometer, the trap detector was compared with a standard silicon detector, 71582 \& serial number: 492, one of the set upon which the scale of detector spectral responsivity was established at NIS (Egypt) and traceable to the cryogenic radiometer.

\subsection{Linearity}

Linearity of the detector was tested using a double beam aperture method ${ }^{[12,13]}$. In this technique the output signal $\mathrm{S}_{\mathrm{a}}$, $\mathrm{S}_{\mathrm{b}}$ are measured for two equal beams $\mathrm{A}$ and $\mathrm{B}$. The sum of these signals, $\mathrm{S}_{\mathrm{a}}+\mathrm{S}_{\mathrm{b}}$ was compared with the signal when both the beams are present in combination, $S_{a b}$. The linearity factor is defined as $\mathrm{S}_{\mathrm{ab}} / \mathrm{S}_{\mathrm{a}}+\mathrm{S}_{\mathrm{b}}$. A wide range of incident power which corresponds to an output current range $A$ to $10^{-4}$ A mpere.

\section{Results and Uncertainties}

\subsection{Luminous Intensity Measurements}

Group of 10 lamps were used, Six FEL 1000 watt and for Wi G Osram 200 watt lamps. The irradiance of the lamp has been measured at discrete wavelengths corresponding to the filters 400,450, 500, 550, 600 and $700 \mathrm{~nm}$. A fitting curve was carried out according to equation 7. The parameters obtained for the lamps from the minimization were considered to be the final results.

From the irradiance distribution, the Illuminance of the lamp at the detector aperture can be determined at known distance d, then calculating the Luminous intensity from equations 2. Five standard lamps operating at color temperature $2856 \mathrm{~K}$ were used. The measurements were performed at a distance of about 2 meters. The luminous intensity resulting from the measurements of the lamps using filtered trap detector was compared to the certified values reported by NPL, as shown in Table (1).

The difference between calibrated lamp at NPL \& the scale based on trap detector illustrated in Figure (4). 


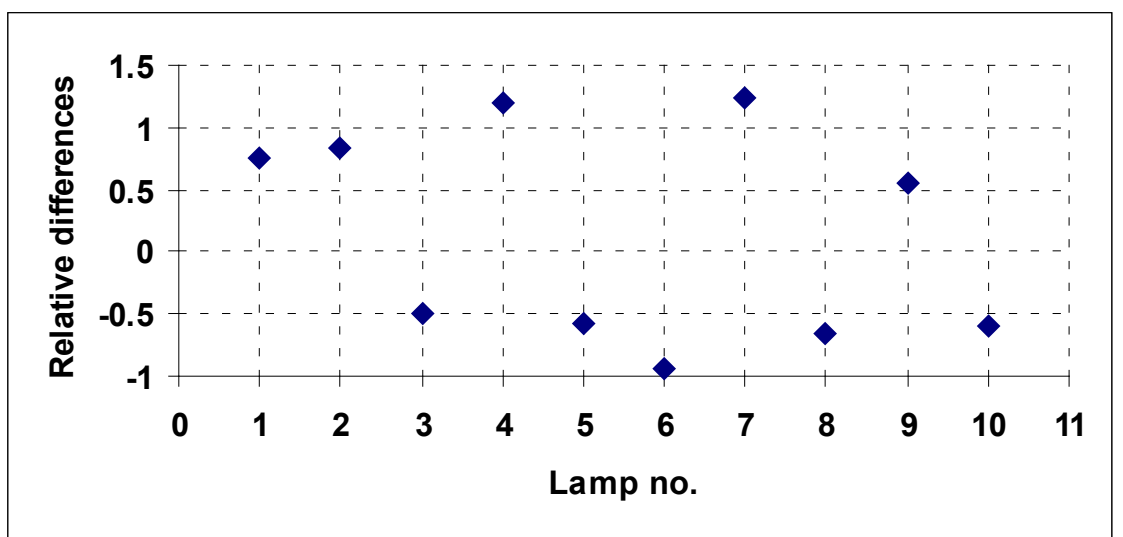

Figure 4. Rat io of the luminous intensity measured by the QED trap detector to the NPL- based value of the lamp

Table.1. Percentage difference between luminous intensity of group of lamps based on trap detector and NPL scale

\begin{tabular}{|c|l|c|c|c|c|}
\hline Lamp no. & \multicolumn{1}{|c|}{ Lamp type } & Current A & $\begin{array}{c}\text { Certified Luminous inten- } \\
\text { sity by NPL in Cd }\end{array}$ & $\begin{array}{c}\text { Measured luminous intensity } \\
\text { by trap detector QED200 }\end{array}$ & $\begin{array}{c}\text { Relative } \\
\text { differences }\end{array}$ \\
\hline 1 & FEL-1000W-BN-9101-420 & 7.34 & 1012.00 & 1019.55 & 0.75 \\
\hline 2 & FEL-1000W-BN-9101-421 & 7.35 & 1055.00 & 1063.82 & 0.84 \\
\hline 3 & FEL-1000W-NIST-2009 & 7.23 & 986.10 & 981.31 & -0.49 \\
\hline 4 & FEL- NIST- F153 & 6.39 & 902.47 & 13.26 & 1.20 \\
\hline 5 & EG-FEL-frosted-NBS-10383 & 7.88 & 1524.00 & 1564.36 & -0.57 \\
\hline 6 & EG-FEL-frosted NBS- 10384 & 7.90 & 1579.00 & 284.70 & -0.94 \\
\hline 7 & Wi41/G Osram- C608-20 & 5.92 & 281.20 & 284.40 & 1.24 \\
\hline 8 & Wi41/G- Osram - C728-43 & 5.91 & 286.30 & 294.35 & -0.66 \\
\hline 9 & Wi41/G-Osram - E13 & 5.99 & 292.70 & 236.57 & 0.56 \\
\hline 10 & Wi41/ G- Osram -E14 & 6.03 & 238.00 & -0.60 \\
\hline
\end{tabular}

Table 2. The individual elements of uncertainty and their contribution to the luminous intensity

\begin{tabular}{|c|c|c|c|}
\hline Component & $10-2 \times$ uncertainty & $\begin{array}{c}\text { Type of uncer- } \\
\text { tainty }\end{array}$ & $\begin{array}{c}10-2 \times \text { relat ive standard uncer- } \\
\text { tainty }\end{array}$ \\
\hline Trap detect or & & & 70 \\
Absolute responsivity at $555 \mathrm{~nm}$ & 140 & $\mathrm{~B}$ & 6.0 \\
Non-linearity 1.2 B & 7 & $\mathrm{~B}$ & 11.5 \\
Spatial non-uniformity 0.5 B & 11 & $\mathrm{~B}$ & 5.8 \\
Filter transmittance & 20 & $\mathrm{~B}$ & 0.6 \\
Transmittance of filter & 10 & $\mathrm{~B}$ & 8.7 \\
Spatial non-uniformity & 1 & $\mathrm{~B}$ & 30 \\
IR leakage 2.0 B & 15 & $\mathrm{~B}$ & 2.7 \\
Measurements & 30 & $\mathrm{~A}$ & 21 \\
Aperture area & 15 & $\mathrm{~B}$ & 6.9 \\
Distance measurements & 28 & $\mathrm{~A}$ & 20 \\
Lamp current & 21 & $\mathrm{~A}$ & 12.7 \\
Alignment repeatability of the lamp & 12 & $\mathrm{~B}$ & 6.9 \\
Alignment repeatability of the filter radiometer & 20 & $\mathrm{~A}$ & 89.8 \\
Stray light & 22 & $\mathrm{~B}$ & 180 \\
Photocurrent measurements & 12 & $\mathrm{~B}$ & \\
Interpolation & & & \\
Interreflection & & & \\
Combined standard uncertainty & & & \\
Expanded uncertainty & & & \\
\hline
\end{tabular}

\subsection{Uncertainties}

The relative uncertainty of the measurements is about $1.8 \%$. The major source of error affecting the uncertainty was the spectral responsivity of the detector. This is due to lower uncertainties in spectral radiometric standards. However, it is possible to reduce the uncertainty, if more accurate spectral responsivity obtained. Also, to minimize errors the trap detector should be temperature-controlled. The diffraction from the aperture and from baffles and the interreflections has a significant effect on the measurements. Table (2) illustrate the ele ments of uncertainty and their contribution to the luminous intensity.

\section{Conclusions}


The SI unit of luminous intensity, the Candela has been realized at National Institute for Standards (NIS) by using a filter radio meter which consis ts of trap detector QED200 and seven interference filters. The detector has been characterized for spectral sensitivity, non-uniformity and linearity to evaluate their stability as irradiance standard. Luminous intensity of group of 10 lamps has been determined by filter radiometry method. These lamps which calibrated at National Physical Laboratory (NPL) with uncertainties of \pm 0.8 were compared with that measured by the trap detector. The comparis on showed an agreement better than $1.8 \%$., which lies within the uncertainty of the two scales.

\section{ACKNOWLEDGEMENTS}

The authors wish to thank Dr Saber Rabie Radiometric Laboratory researcher to provide assistance to us.

\section{REFERENCES}

[1] BIPM Comptes Rendus 16th Conf. G'en. Poids et Mesures, S'evres, Bureau International des Poids et Mesures, 1979, 100; Giacomo P., Metrologia, 1980, 16, 55-61; Giacomo P., Metrologia, 1981, 17, 74.

[2] The Basis of Physical Photometry, CIE Publ. No. 18.2, Vienna, International Commission on Illumination, 1983, 42 p.

[3] Zalewski E. F., Duda C. R., Appl. Opt., 1983, 22, 2867-2873.

[4] Fox N. P., Trap detectors and their properties, Metrologia, 1991, 28, 197-202.

[5] Durak M, Samedov F: Realization of a filter radiometer based irradiance scale with high accuracy in the region of $286 \mathrm{~nm}$ to $901 \mathrm{~nm}$. Metrologia, 2004, 39,(6)
[6] Farhad Samedov, Murat Durak, Özcan Bazkır Filter-radiometer-based realization of candela and establishment of photometric scale at UME Original Research Article, Optics and Lasers in Engineering,43,11, 2005, 1252-1266

[7] Applied Photometry, Radiometry, And Measurements Of Optical Losses, Springer Series in Optical Sciences, Vol., $163,2012,49-128$.

[8] Yoshihiro Ohno, Improved Photometric Standards and Calibration Procedures at NIST J. Res. Natl. Inst. Stand. Technol. 102(1997) 323.

[9] F. Sametoglu, New traceability chains in the photometric and radiometric measurements at the National Metrology Institute of Turkey Optics and Lasers in Engineering, 2007, 45, $1,36-42$.

[10] Andor G: New data-reduction method in detector-based spectral-irradiance measurements. Metrologia, 1996, 32, 495-496

[11] Özcan Bazkır, Farhad Samadov, Characterization of silicon photodiode-based trap detectors and establishment of spectral responsivity scale, Optics and Lasers in Engineering, 43,2, 2005, 131-141

[12] Thompson, A. and Chen, H., " Beamcon III , a lineartity measurement for optical detectors," J.Res. NIST, 1994, 99(6), 751-755

[13] M Noorma1, P Toivanen, F Manoocheri and E Ikonen, Characterization of filter radiometers with a wavelength-tunable laser source Metrologia, 2003, 40 S220-S223, Optics and Lasers in Engin eering, 43,2, 2005, 131-141

[14] Ikonen E.; Karha P.; Lassila A.; Manoochehri F.; Fagerlund H.; Liedquist L.m , Radiometric realization of the candela with a trap detector,32, 6, 1995, pp. 689

[15] F Sametoglu, "Evaluation of expanded uncertainties in luminous intensity and illuminance calibrations", Applied optics, 47, 31, 2008, 5829-5847. 